\title{
Strength, efficacy and tolerability of morphine solution with and without added preservatives: A comparative study
}

\section{Rawshan Ara Perveen and Md. Sayedur Rahman}

Department of Pharmacology, Bangabandhu Sheikh Mujib Medical University, Shahbag, Dhaka, Bangladesh.

\begin{tabular}{|c|c|}
\hline \multicolumn{2}{|l|}{ Article Info } \\
\hline Received: & 27 January 2014 \\
\hline Accepted: & 15 February 2014 \\
\hline Available Online: & 18 February 2015 \\
\hline \multicolumn{2}{|c|}{ DOI: 10.3329/bjp.v10i1.21754 } \\
\hline \multicolumn{2}{|c|}{$\begin{array}{l}\text { Cite this article: } \\
\text { Perveen RA, Rahman MS. Strength, } \\
\text { efficacy and tolerability of morphine } \\
\text { solution with and without added } \\
\text { preservatives: A comparative study. } \\
\text { Bangladesh J Pharmacol. 2015; } 10: 116 \\
-24 \text {. }\end{array}$} \\
\hline
\end{tabular}

\begin{abstract}
Cancer patients having moderate to severe pain need immediate pain relief. Immediate release formulation of morphine is not available in Bangladesh. This open label randomised two phase crossover clinical trial was conducted to compare strength, efficacy, and tolerability of oral morphine solution prepared from morphine sulfate injectable formulation and sustained release tablet with and without added preservatives. Concentration of morphine in solutions both with and without added preservative was highest on the first day and then gradually decreased. No difference between two storage temperatures on first day, but highly significant difference afterwards. Rate of degradation of morphine in solution with added preservative was significantly slower. The present study revealed that the solutions prepared from sustained release tablets and injectable formulation with added preservative could be an option to control severe pain in cancer patients. To introduce the present research finding in clinical practice, this has to be accepted institutionally.
\end{abstract}

\section{Introduction}

Morphine has been accepted as gold standard drug of choice to treat moderate to severe pain in advanced cancer (WHO, 1996). Oral route is the simplest, the most acceptable and preferable route. Aqueous solutions of morphine sulfate or hydrochloride salt and tablet or capsule preparation is recommended for oral administration. Immediate release tablet, also known as soluble tablet is cheap, useful for dose titration and quick effect. On the other hand, modified or slow release formulation is recommended for maintenance treatment though relatively expensive has longer duration of action (Hanks et al., 2001).

Regarding availability of morphine, restrictive narcotic law of Bangladesh has contributed negatively. In addition, awareness of health care professionals about use of Morphine in effective pain control is still inadequate (Khatun et al., 2008; Dehgan et al., 2010). Moreover, in Bangladesh, due to complicated regula- tory formalities and less profit opportunity, morphine preparation has been manufactured by only one pharmaceutical company, injectable preparation (G Morphine) and sustained release tablet formulation ( $G$ Morphine SR) are available and both contained $15 \mathrm{mg}$ morphine sulfate. Immediate release formulation of morphine either as tablet or as solution is not available in Bangladesh (Dehgan et al., 2010).

Cancer patients having moderate to severe pain need immediate pain relief. Immediate release formulation is also ideal for breakthrough pain. Some patients consider solution form more convenient to consume. In the background of this prevailing reality in Bangladesh, Centre for Palliative Care, Bangabandhu Sheikh Mujib Medical University prepared an immediate release morphine solution from injectable formulation of morphine sulfate with distilled water.

In the present study, morphine solution from sustained release tablet was prepared, which was comparatively 
cheaper than injectable formulation and modified both formulations by adding more preservative. This open label randomised two phase crossover clinical trial was conducted to compare the strength, efficacy, and tolerability of oral morphine solution prepared from morphine sulfate injectable formulation and sustained release tablet with and without added preservatives in cancer patients.

\section{Materials and Methods}

\section{Preparation of solution}

Four different types of morphine solutions were prepared. One solution was prepared from morphine sulfate injectable formulations with deionized water. Another solution was prepared from morphine sulfate injectable formulations with deionized water and preservative was added and finally stored in an amber glass container. Preservatives used were sodium metabisulfite $(0.9 \mathrm{mg})$, sodium chloride $(7.6 \mathrm{mg})$, sodium citrate $(0.2 \mathrm{mg})$ and citric acid $(0.4 \mathrm{mg})$ for $1 \mathrm{mg}$ morphine sulfate stored in an amber glass container (British Pharmacopeia, 2009).

Solutions prepared from sustained release tablets were dissolved for 20 hours in deionized water in room temperature $\left(20-25^{\circ} \mathrm{C}\right)$, then the solutions was filtered with filter paper and deionized water was added and stored in an amber glass container. Another solution was prepared from sustained release tablets following same procedure and preservative was added and mixed with filtered solution in an amber glass container.

These solutions were stored into amber glass bottles in equal amount and kept at $4^{\circ} \mathrm{C}$ and room temperature $\left(20-25^{\circ} \mathrm{C}\right)$ (Preechagoon et al., 2005). Then morphine concentration was estimated in solution in the day just after preparation and then every 24 hour after up to 96 hours of storage.

Each day $20 \mu \mathrm{L}$ of stored solution and $980 \mu \mathrm{L}$ of $0.1 \mathrm{M}$ $\mathrm{NaOH}$ solution was added in a test tube and covered with aluminum foil paper till estimation. The absorbance was estimated by the spectrophotometer at 298 $\mathrm{nm}$ wavelength against reagent blank. The reading was adjusted at 0.000 in the spectrophotometer by using $0.1 \mathrm{M} \mathrm{NaOH}$ before measurement (British Pharmacopeia, 2009).

\section{Subject inclusion}

Patient with advanced cancer who mentioned pain intensity more or equal to 7 in the Visual Analogue Scale (VAS) were included in the study. Patient who received chemotherapy for last 15 days and unable to communicate verbally were excluded from the study. Thirty six admitted cancer patients who received at least single dose of morphine solution were considered as Intent to Treat (ITT) population and were divided into two groups. One group consists of 17 patients, who received solutions prepared from morphine without added preservatives. Another group consisted of 19 patients received solutions prepared with added preservatives. Among them 4 patients from each group, i.e., total 8 patients could not complete the study for different reasons. Therefore, 28 patients who completed the study were considered as per protocol population.

Before assessment of pain intensity, VAS was explained to each patient, who fulfilled the inclusion criteria. In VAS, 0 means no pain and 10 means worst possible pain (Scott and Huskisson, 1979). In first phase of the study, after explanation of VAS, VAS score was taken before morphine solution. Then after $0.3,0.6,1,1.5$ and 4 hours of administration morphine solution, patient was asked about his pain intensity. In second phase of the study, pain intensity of patients was recorded after 1.5 hours of $1^{\text {st }}, 2^{\text {nd }}, 3^{\text {rd }}$ and $4^{\text {th }}$ doses of administration. Number in the scale mentioned by patient was recorded in data collection sheet.

In first phase, patient who received morphine solution without added preservative from sustained release tablet from $1^{\text {st }}$ to $4^{\text {th }}$ day of the study, crossed over with solution prepared by injectable formulation without any washout period from $5^{\text {th }}$ to $8^{\text {th }}$ day of the study. Patients who received morphine solution prepared by injectable formulation from $1^{\text {st }}$ to $4^{\text {th }}$ day of the study crossed over with the solution prepared with sustained release tablets from $5^{\text {th }}$ to $8^{\text {th }}$ day of the study. After completion of the study with morphine solution without added preservative group, then study started with added preservative group in same manner.

Both groups of patients were administered morphine solution orally after assessing their pain intensity by VAS. After $20 \mathrm{~min}$ of administration, the intensity of pain was reassessed by VAS and relief of pain by pain relief score. During this period, patients were asked about side effects and assessed by 4 point categorical scale. After $40 \mathrm{~min}, 1$ hour, 1.5 hour and 4 hours of administration, VAS, pain relief score and side effects score were recorded for next seven days once daily over a 4 hours period after drug administration.

In second phase of the study, one group of patients received solutions prepared by sustained release tablets without added preservative and another group received solutions with added preservative, both group for one day. Patients were administered morphine solution orally after assessing their pain intensity by VAS. Then $1^{\text {st }}$ dose of morphine solution was administered and pain intensity was recorded after 1.5 hours by VAS. Then pain intensity of patients was recorded after 1.5 hours of $2^{\text {nd }}, 3^{\text {rd }}$ and $4^{\text {th }}$ doses of administration.

Patient who complained breakthrough pain in both phases, treated with morphine solution as rescue doses (Hagan et al., 2008). Frequency of administration and 
amount of rescue doses were also recorded.

Opioid naive patients received oral morphine solution 5 mg every 4 hours as the starting dose (irrespective of injectable formulation and sustained release tablet). Opioid tolerable patients received $10 \mathrm{mg}$ every 4 hours was the starting dose (Hanks et al., 2001) and by morphine trial (Kumar et al., 2000).

Morphine solutions were administered at 8:00 am, 12:00 pm, 4:00 pm, 8:00 pm and 12:00 pm at local time (Hanks et al., 2001). A double dose was administered at bedtime to avoid nocturnal dosing (Dale et al., 2009; Todd et al., 2002). Every day of study total daily dose of morphine solution was adjusted according to the patient's need (WHO, 1996). Total daily doses were increased if patients complained and decreased if side effect especially somnolence was developed. No maximal dosage limit was imposed by the protocol, unless any uncontrollable adverse event aroused.

Patients who experienced breakthrough pain during the interval between two regular doses took rescue doses of oral morphine solution. Rescue doses were the same as the patient's regular doses morphine solution (Zeppetella, 2009). The total daily dosage was titrated on patients who took that twice and more rescue doses of solution in previous 24 hours along with his/her regular dose of solution (Hanks et al., 2001).

In first phase, mean pain intensity was calculated before and after administration morphine solution. In second phase, mean pain intensity were calculated before and after patient received $1^{\text {st }}, 2^{\text {nd }}, 3^{\text {rd }}$ and $4^{\text {th }}$ dose of solutions.

Before assessment of pain relief score, each patient who fulfilled the inclusion criteria was asked about his pain relief after administration of morphine solution. Pain relief score was assessed after administration of morphine solution. Qualitative data mentioned by patient was interpreted by researcher that if patient replied 0 means pain was not relieved after drug administration, little relief of pain interpreted as 1, some relief of pain interpreted as 2, a lot relief of pain interpreted as 3 and complete relief of pain interpreted as 4 (Likert, 1932). All data were then recorded in data collection sheet.

Each patient included in the study, had to reply their pain relief maximum at 4 in one single assessment. Similar assessment done after 0.3, 0.6, 1, 1.5 and 4 hours. Therefore, in this time period individual patient had to reply his pain relief on five occasions and consequently a maximum at 20 in a single day of study. In a four day study, individual patient had to reply his pain relief score to a maximum of 80 . The summative total pain relief score was calculated among the per protocol patient in each group. Then the cumulative pain relief score as replied by individual per protocol patient in a single day was calculated. Then their pain relief score in four day study was calculated for an individual patients received solutions prepared with sustained release tablets or injectable solutions with and without added preservative.

In side effect score, patient was assured that he could mention more than one side effect. Each of the side effects was assessed separately at 0.3, 0.6, 1, 1.5 and 4 hours after morphine solution administration. Complete absence of any side effects was interpreted as 0 , mild form of side effects interpreted as 1, moderate form of side effects was interpreted as 2 and severe form of side effects interpreted as 3 (Dawnie et al., 1978). Number of the scale was interpreted by the researcher and later was recorded in data collection sheet.

Each patient reported in the study, had to reply their side effects as severe at 3 , moderate at 2 and mild at 1 in one single assessment. Side effects scores were recorded after administration of solution and finally mean side effects daily was calculated. In each 4 day phase, individual patient might have replied his severe form of side effects as 12, moderate form as 8 and mild form as 4 for a single adverse effect. Then calculated the cumulative side effects score replied by individual per protocol patient in different groups. Each side effect was compared with highest mild, moderate and severe form side effects score versus cumulative score of per protocol patient of each group.

\section{Result}

Morphine sulfate sustained release tablets dissolved in deionized water shows different concentration of morphine $(\mu \mathrm{g})$ in filtered solutions at different time. Highest concentration of morphine $(1398.7 \mu \mathrm{g})$ was found in solution after 20 hours of dissolution (Table I).

Figure 1 shows the concentration of morphine in solution prepared from sustained release tablets and injectable formulation of morphine sulfate without and with added preservative, stored in room temperature $\left(20-25^{\circ} \mathrm{C}\right)$ and $4^{\circ} \mathrm{C}$ temperature. Concentration of mor-

\begin{tabular}{|cc|}
\hline \multicolumn{2}{|c|}{ Table I } \\
\hline Dissolve test of sustained release morphine sulfate \\
tablets
\end{tabular}

Values are expressed as mean $\pm S D ; n=6$ 


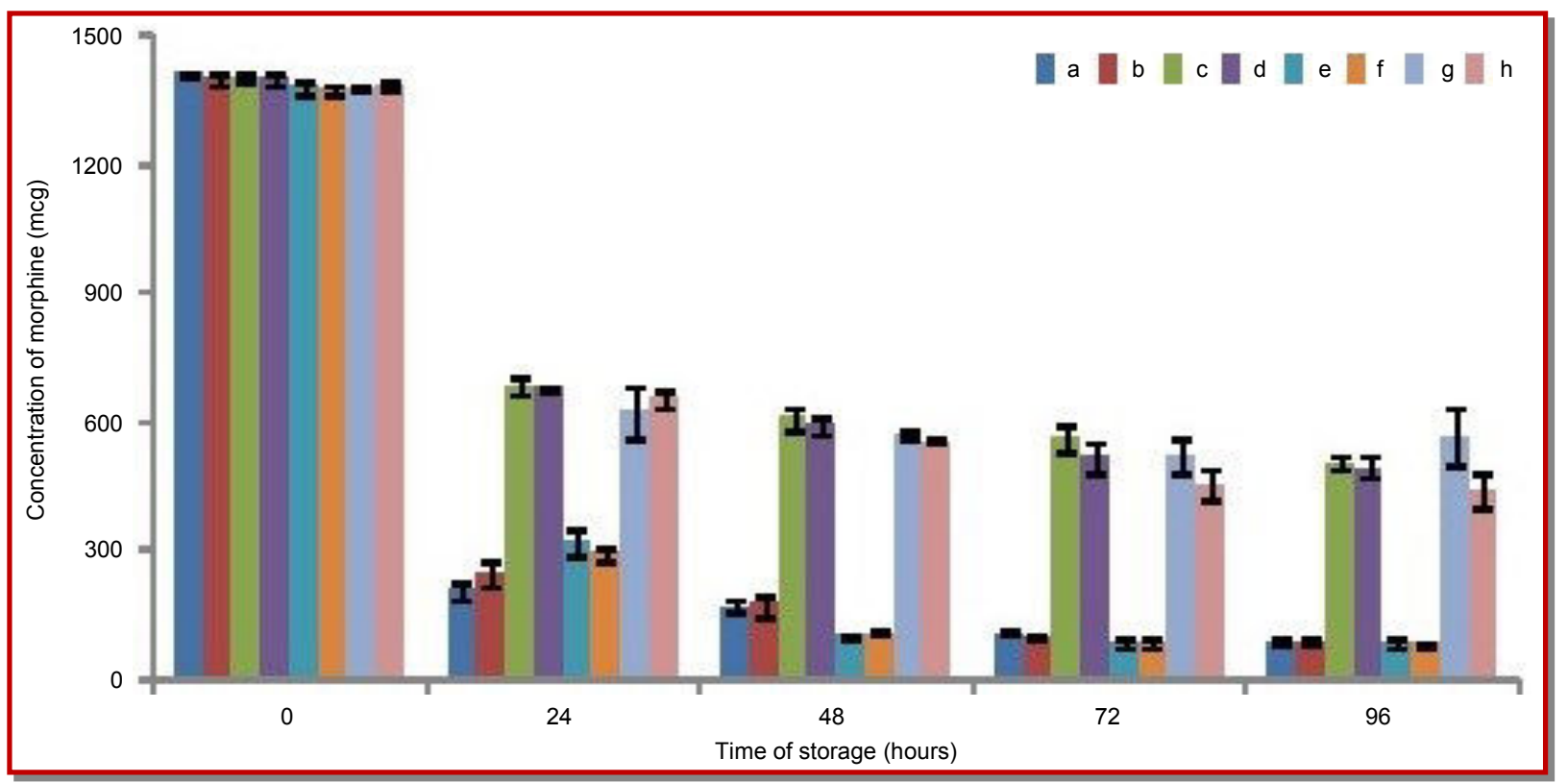

Figure 1: Estimation of morphine in solution prepared from sustained release tablets and injectable formulation without and with added preservative stored at different temperature and time

Solution prepared by sustained release tablet- without (a) or with (b) added preservative stored in room temperature $\left(20-25^{\circ} \mathrm{C}\right)$; without $(\mathrm{c})$ or with (d) added preservative stored $4^{\circ} \mathrm{C}$ temperature. Solution prepared by injectable formulation - without (e) or with (f) added preservative stored in room temperature $\left(20-25^{\circ} \mathrm{C}\right)$; without $(\mathrm{g})$ or with $(\mathrm{h})$ added preservative stored $4^{\circ} \mathrm{C}$ temperature

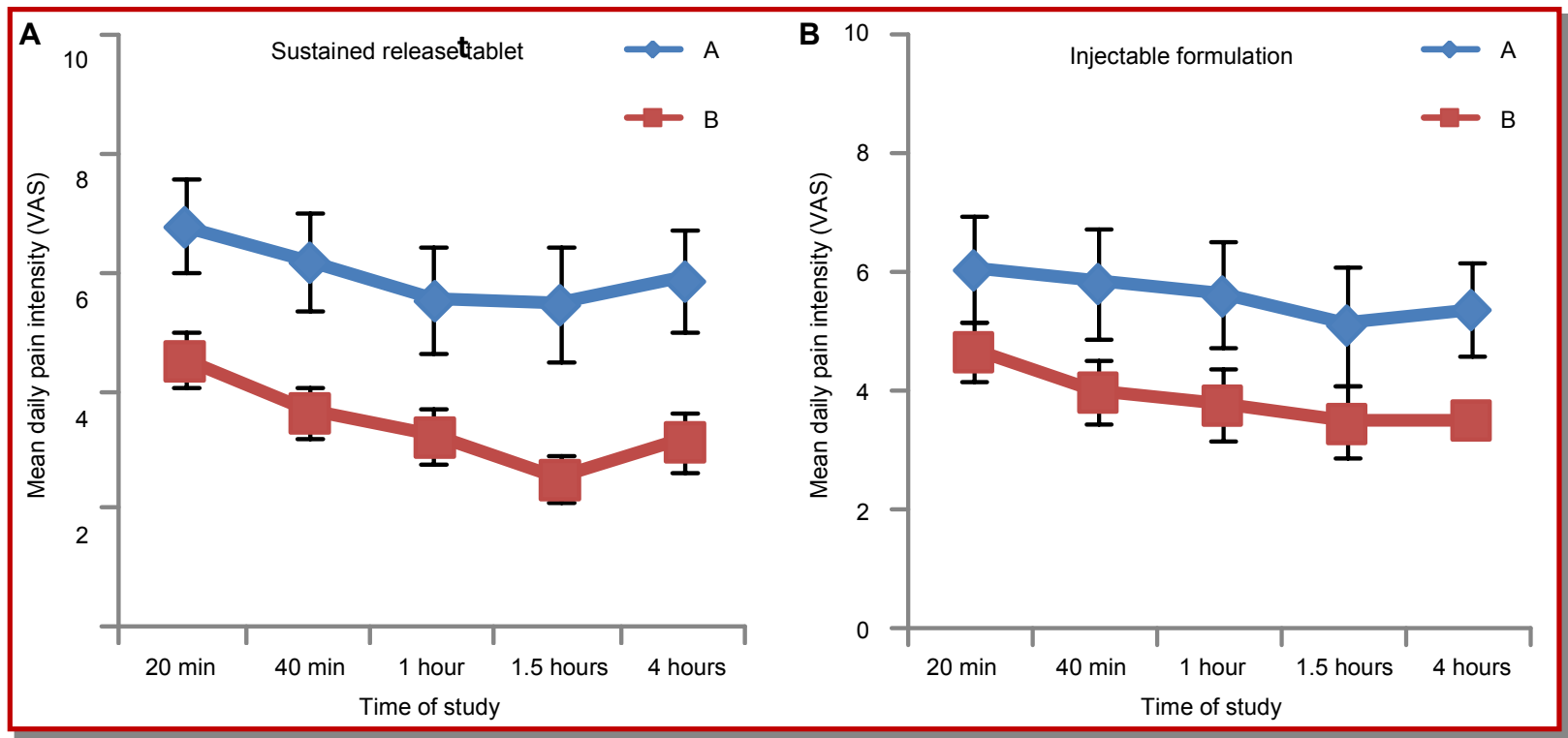

Figure 2: Differences in Mean Daily Pain Intensity after administration of morphine solution prepared by sustained release tablet (A) and injectable formulation (B) without and with added preservative over a four hour study period

A: solution prepared without added preservative; B: solution prepared with added preservative

phine was highest on first day of the study just after preparation of solutions in both temperatures. Then concentration of morphine had gradually decreased on second, third, fourth and fifth day.

'Paired $t^{\prime}$ test was done. No significant difference found on first day of study of two groups. Highly significant difference found in solution on second, third, fourth and fifth day of study in solutions with and without added preservative. No significant difference was found on first, second, third, fourth and fifth day among solutions without added preservative but significant difference was found on second, third, fourth and fifth day among solutions with added preservative.

Figure 2 shows mean daily pain intensity changes according to visual analogue scale within four hours 


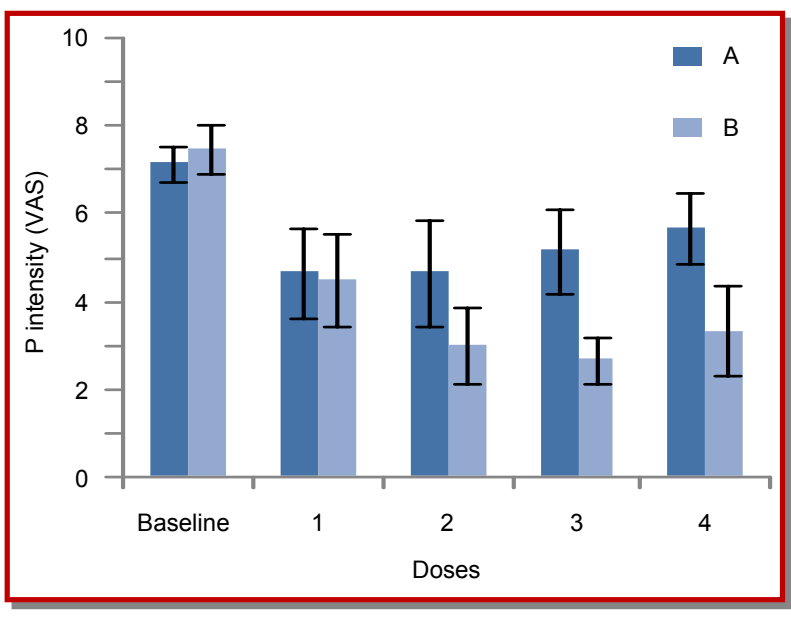

Figure 3: Differences in pain intensity after administration of solution prepared by sustained release tablets with and without added preservatives in a day

$\mathrm{x}$ : solutions prepared without added preservative; $\mathrm{y}$ : solutions prepared with added preservative

period of study. Solution prepared by sustained release tablets without added preservative highest mean daily pain intensity \pm SD at $20 \mathrm{~min}$ after administration. Values were gradually decreasing at $40 \mathrm{~min}, 1$ hour, 1.5 hour after administration. Lowest pain intensity recorded at 1.5 hour over a four hour study period. Then at 4 hours after administration of solution mean daily pain intensity slightly increase from previous records. Solution prepared with added preservative shows same result. Same result of mean daily pain intensity changes shows after administration of morphine solution prepared by Injectable formulation without and with added preservative over a four hour study period.

Figure 3 shows changes of pain intensity in visual analogue scale over a day after 1.5 hours of morphine solution administration. Pain intensity marked as baseline, before administration of solution prepared by sustained release tablets without added preservative was highest, after 1.5 hours of $1^{\text {st }}$ dose of morphine solution administration was decreased, when patient received solution just after preparation. After $2^{\text {nd }}$ dose intensity was also decreased, when solution was stored for 4 hours. After 8 hours storage $3^{\text {rd }}$ dose of solution and after 12 hours of storage at $4^{\text {th }}$ dose was administered, intensity were slightly increased from previous records.

Pain intensity before administration of solution prepared by sustained release tablets with added presservative was highest. Pain intensity after 1.5 hours of $1^{\text {st, }}$ $2^{\text {nd }}, 3^{\text {rd }}$ and $4^{\text {th }}$ dose of morphine solution administration was gradually decreased. 'Wilcoxon signed rank' test was done. Significant difference was found between two solutions.

Figure 4 shows total pain relief score of patients

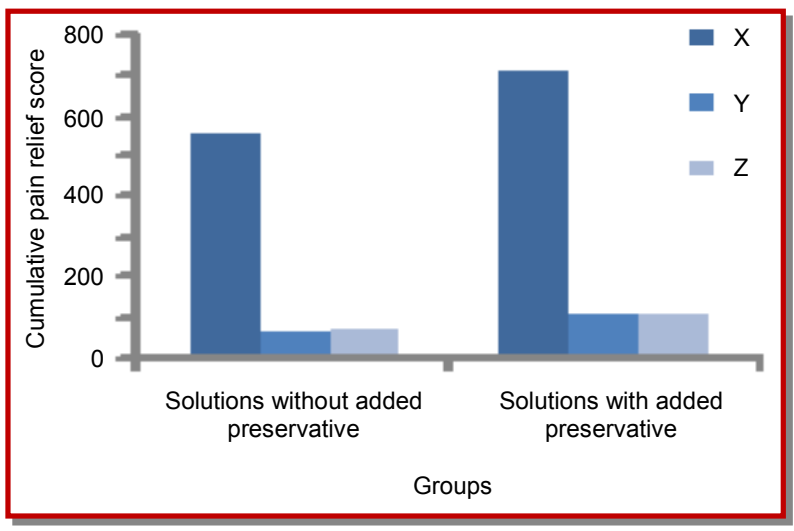

Figure 4: Cumulative pain relief score after administration of morphine solution prepared from sustained release tablets and injectable formulations with and without added preservative

$\mathrm{x}$ : Total pain relief score of $7(80 \times 7)$ patients included in solutions without added preservative and $9(80 \times 9)$ patients included in solutions with added preservative; y: Cumulative pain relief score after morphine solution administration prepared from sustained release tablets; z: Cumulative pain relief score after morphine solution administration prepared from injectable formulation

received solutions without added preservative was 560 . Cumulative pain relief score after administration of solution prepared with sustained release tablets was 71 and with injectable formulation was 72 . Total pain relief score of patients received solutions with added preservative was 720 . Cumulative pain relief score after administration of solution prepared with sustained release tablets was 110 and injectable formulation was 116. 'Wilcoxon signed ranks' test was done. No significant difference found on solutions prepared by sustained release tablets and injectable formulations with vs without added preservative.

Table II and III shows cumulative side effect score. Kruskal wallis test was done. No significant difference was found in solutions prepared with sustained release tablets and injectable solutions with vs without added preservation.

\section{Discussion}

Concentration of morphine in solutions both with and without added preservative was highest on the first day. Then in both cases, concentrations were gradually decreased on second, third, fourth and fifth day of stored solutions both in room temperature $\left(20-25^{\circ} \mathrm{C}\right)$ and $4^{\circ} \mathrm{C}$ temperatures. No significant difference was found between two storage temperature in all solutions on first day, but highly significant difference was found at second, third, fourth day of storage. The rate of degradation of morphine in solution with added preservative was significantly slower in comparison with solutions without added preservative. Higher rate of degradation of morphine in solutions without added preservatives occurred possibly due to formation of morphine-n-oxide, pseudomorphine and apomorphine. 


\section{Table II}

Cumulative side effects score after administration of morphine solution prepared with morphine sulphate sustained release tablets with and without added preservative

\begin{tabular}{|c|c|c|c|c|c|c|c|}
\hline \multirow[t]{3}{*}{ Side effects } & \multicolumn{6}{|c|}{ Cumulative side effects score } & \multirow[t]{3}{*}{$\mathrm{p}$} \\
\hline & \multicolumn{3}{|c|}{$\begin{array}{l}\text { Sustained release tablets without added pre- } \\
\text { servative }(n=7)\end{array}$} & \multicolumn{3}{|c|}{$\begin{array}{l}\text { Sustained release tablets with added preserv- } \\
\text { ative }(n=9)\end{array}$} & \\
\hline & Mild & Moderate & Severe & Mild & Moderate & Severe & \\
\hline Constipation & $09 / 28$ & $08 / 56$ & $00 / 84$ & $10 / 28$ & $04 / 56$ & $00 / 84$ & \multirow[t]{9}{*}{0.816} \\
\hline Drowsiness & $08 / 12$ & $00 / 24$ & $00 / 84$ & $07 / 32$ & $09 / 64$ & $03 / 96$ & \\
\hline Nausea & $07 / 08$ & $00 / 16$ & $00 / 24$ & $02 / 16$ & $00 / 32$ & $00 / 48$ & \\
\hline Vomiting & $01 / 08$ & $00 / 16$ & $00 / 24$ & $03 / 16$ & $02 / 32$ & $00 / 48$ & \\
\hline Fever & $01 / 08$ & $00 / 16$ & $00 / 24$ & $05 / 08$ & $00 / 16$ & $00 / 24$ & \\
\hline Restlessness & $01 / 04$ & $04 / 08$ & $03 / 12$ & $02 / 08$ & $04 / 16$ & $00 / 24$ & \\
\hline Burning sensation & $00 / 04$ & $00 / 08$ & $12 / 12$ & $01 / 04$ & $06 / 08$ & $00 / 12$ & \\
\hline Diarrhoea & $01 / 04$ & $00 / 08$ & $00 / 12$ & $01 / 04$ & $00 / 08$ & $00 / 12$ & \\
\hline Weakness & $00 / 04$ & $02 / 08$ & $00 / 12$ & $01 / 04$ & 06/08 & $00 / 12$ & \\
\hline
\end{tabular}

\section{Table III}

\section{Cumulative side effects score after administration of morphine solution prepared with morphine sulphate} injectable formulation with and without added preservative

\begin{tabular}{|c|c|c|c|c|c|c|c|}
\hline \multirow[t]{3}{*}{ Side effects } & \multicolumn{6}{|c|}{ Cumulative side effects score } & \multirow[t]{3}{*}{$\mathrm{p}$} \\
\hline & \multicolumn{3}{|c|}{$\begin{array}{l}\text { Injectable formulation without added pre- } \\
\text { servative }(n=7)\end{array}$} & \multicolumn{3}{|c|}{$\begin{array}{l}\text { Injectable formulation with added preserva- } \\
\text { tive }(\mathrm{n}=9)\end{array}$} & \\
\hline & Mild & Moderate & Severe & Mild & Moderate & Severe & \\
\hline Constipation & $8 / 24$ & $6 / 56$ & $3 / 84$ & $4 / 28$ & $8 / 56$ & $15 / 84$ & \multirow[t]{9}{*}{0.894} \\
\hline Drowsiness & $1 / 12$ & $8 / 24$ & $0 / 48$ & $13 / 32$ & $0 / 64$ & $9 / 96$ & \\
\hline Nausea & $2 / 8$ & $0 / 16$ & $0 / 24$ & $2 / 16$ & $0 / 32$ & $0 / 48$ & \\
\hline Vomiting & $2 / 8$ & $0 / 16$ & $0 / 24$ & $4 / 16$ & $0 / 32$ & $0 / 48$ & \\
\hline Fever & $0 / 8$ & $2 / 16$ & $0 / 24$ & $0 / 08$ & $0 / 16$ & $0 / 24$ & \\
\hline Restlessness & $1 / 4$ & $2 / 8$ & $0 / 12$ & $0 / 8$ & $0 / 16$ & $0 / 24$ & \\
\hline Burning sensation & $0 / 4$ & $0 / 8$ & $9 / 12$ & $0 / 4$ & $0 / 8$ & $0 / 12$ & \\
\hline Diarrhoea & $0 / 4$ & $0 / 8$ & $0 / 12$ & $0 / 4$ & $0 / 8$ & $0 / 12$ & \\
\hline Weakness & $0 / 4$ & $0 / 8$ & $0 / 12$ & $0 / 4$ & $8 / 8$ & $0 / 12$ & \\
\hline
\end{tabular}

Morphine usually produces these metabolites when it comes in contact with atmospheric oxygen. Yeh and Lach (1961) first mentioned that degradation of morphine in solutions occurred in presence of atmospheric oxygen in the system. Vermeire and Remon (1999) concluded that degradation of morphine in aqueous solution is accelerated in the presence of atmospheric oxygen with the formation of mainly pseudomorphine, to a lesser extent morphine-n-oxide and probably apomorphine. Oxidation reaction was considered as the major problem related to storage of morphine solution.

Solutions prepared with added preservative that contain sodium metabisulphite, sodium citrate, citric acid and sodium chloride (British Pharmacopeia, 2009) showed slower degradation of morphine in solutions. Sodium metabisulphite is an established antioxidant and reported to prevent oxidation of morphine. Couple of researchers added sodium metabisulphite to prevent degradation of morphine from oxidation reaction and sodium citrate to improve test without affecting stability of oral morphine solution in their study. Possibly due to presence of sodium metabisulphite in the preservative used in the present study, degradation rate of morphine was slower (Grassby and Hutchings, 1993; Preechagoon et al., 2005).

No significant difference was observed between solutions irrespective of method of preparation and pre- 
sence of added preservative when stored in room temperature $\left(20-25^{\circ} \mathrm{C}\right)$. Vermeire and Remon (1999) showed earlier that temperature had a minor influence on the degradation rate of morphine and recommended that morphine solutions should preferably be stored at room temperature. Lawrence et al., (2002) found concentrations of morphine in solutions were stable at $4^{\circ} \mathrm{C}$ and $23^{\circ} \mathrm{C}$. Lee et al. (2003) showed morphine solutions stored at room temperature and at $4^{\circ} \mathrm{C}$ had no visual evidence of discoloration, precipitation or cloudiness over the 14 days test period. The findings of the present study are consistent with previous study results.

The demographic characteristics data of intent to treat population demonstrate that among the malignancies, carcinoma of breast and stomach cancer were highest and there was extensive diversity in pattern of malignancies as reflected by 20 types of cancer in such a small study. Equal number of male and female patients was observed. An average of two years' time was elapsed after the study subjects got the diagnosis while they were receiving palliative care service.

The present study revealed that, no significant difference was found between mean daily pain intensity between solutions prepared from sustained release tablets and injectable formulations with and without added preservative, when pain intensity was recorded just after preparation. But significant difference in pain intensity was found after addition of additional preservative in morphine solutions after several hours of storage.

Arkinstall et al., (1989) showed that administration of oral morphine solution in 4 hours interval effectively controls severe pain. Previous study showed decreased mean daily pain intensity up to $1.4 \pm 0.2$ in immediate release morphine in patients with moderate to severe cancer pain (Deschamps et al., 1992). MERITO study shows that immediate release oral morphine effectively and rapidly decrease the pain intensity after 5 days in cancer patient with moderate to severe pain (Conno et al., 2008). Eyelade et al., (2012) mentioned that pretreatment pain intensity score reduced 3 point at the end of the first week of treatment with an immediate release oral morphine elixir.

The present study shows no significant difference in case of pain relief after administration of solutions prepared from sustained release tablets and injectable formulation through pain relief score. This one dimensional scale reflected pain perception of patients, which had some limitations like patients avoided extreme positive responses. The 11-point (0-10) visual analogue scale performs better than both a 4-point simple descriptive scale (Downie et al., 1978). The present study also supports this statement.

Various side effects like constipation, drowsiness, nausea, vomiting, fever, restlessness, burning sensation, diarrhoea and restlessness were reported. No significant difference was found after administration of solutions prepared by sustained release tablets and injecttable formulations with and without preservative.

Low level of nausea, confusion and drowsiness after of morphine solution was recorded (Melzack et al., 1979). They also mentioned in their study that 37\% patients reported side effects like constipation, nausea, vomiting and somnolence for more than four days. Finn et al., (1993) mentioned sedation, nausea, anxiety and depression after administration of immediate release morphine sulfate solution. Somnolence, nausea, constipation and dizziness were the most common morphine associated side effects after administration of morphine sulfate immediate release formulation (Colluze et al., 2001). Quigly (2003) assessed side effects of morphine by side effects score in patients with cancer related pain in their study. $10.9 \%$ constipation, $22.8 \%$ nausea, $18.8 \%$ vomiting, $10.9 \%$ somnolence, $3 \%$ weakness, $1 \%$ diarrhoea and pyrexia after administration of immediate release morphine was reported earlier (Christensen et al., 2008). Dale et al., (2009) reported constipation, nausea, xerostomia after administration of immediate release oral morphine. Kamuhabwa and Ezekiel, (2009) mentioned that 79\% patient experienced morphine induced side effects, of which $73 \%$ reported constipation, 31\% reported vomiting, 30\% reported nausea and $24 \%$ reported drowsiness.

In order to correlate the pharmacological effects with newer formulation, understanding about the pharmacokinetics of newly prepared formulation needs to be evaluated. The pharmacokinetic status of Bangladeshi population is still not very clearly understood, though couple of studies revealed that orally administered medicines achieve adequate concentration to demonstrate desired pharmacological effects (Chowdhury et al., 2010; Chowdhury et al., 2011; Chowdhury et al., 2014). The present study was not designed to ascertain or clarify the correlation of kinetics with dynamics.

Among different limitations, the study could not be executed as double blind study because of the unavailability of morphine. Furthermore, both groups could not run in parallel and variation in dose of morphine for individual patient could not be kept uniform.

This can be presumed from the present study that the solutions prepared from sustained release tablets and injectable formulation with added preservative could be an alternative to control severe pain in cancer patients of Bangladesh. Nevertheless, research findings are usually not translated into clinical practice in Bangladesh as revealed in different previously conducted studies (Khatun et al., 2008; Rahman and Huda, 2014; Afreen and Rahman, 2014). The present undergraduate and postgraduate medical education programs are not responsive or accommodative to newer 
research findings and approaches (Rahman, 1995; Begum et al., 1999; Rahman et al., 2000). Therefore, in order to introduce the present research finding in clinical practice, this knowledge has to be accepted institutionally as well as should be incorporated formally into the courses of relevant postgraduate disciplines through innovative and interesting approach.

\section{Acknowledgements}

We gratefully acknowledge the laboratory supports provided by the Department of Pharmacology and patients related supports by the Center for Palliative Care, Bangabandhu Sheikh Mujib Medical University.

\section{References}

Afreen S, Rahman MS. Adherence to treatment guidelines in a university hospital: Exploration of facts and factors. Bangladesh J Pharmacol. 2014: 9: 182-88.

Arkinstall WW, Goughnour BR, White JA, Stewart JH. Control of severe pain with sustained-release morphine tablets $\mathrm{v}$. oral morphine solution. CMAJ. 1989; 140: 653-61.

Begum M, Rahman MS, Islam AFMS, Khan IA, Akhter N. Eleven years of the undergraduate medical curriculum 1988: Review on the changes in pharmacology written questions. Bangladesh J Physiol Pharmacol. 1999: 15: 27-30.

British Pharmacopeia commission 2009, British Pharmacopeia, the stationary office, United Kingdom.

Chowdhury RZ, Islam MS, Rahman MS. Pharmacokinetic parameters of amoxicillin in Bangladeshi volunteers: A preliminary evaluation. Bangladesh J Physiol Pharmacol. 2010: 26: 1-9.

Chowdhury RZ, Islam MS, Rahman MS. Pharmacokinetic parameters of ciprofloxacin in Bangladeshi volunteers: A preliminary evaluation. Bangladesh J Physiol Pharmacol. 2011: 27: 1-8.

Chowdhury RZ, Islam MS, Rahman MS. Pharmacokinetic study after single oral dose of beta-lactam and fluoroquinolone antimicrobials in Bangladeshi healthy male volunteers. Bangladesh J Physiol Pharmacol. 2014: 30: 16-24.

Christensen KS, Cohen AE, Mermelstein FH, Hamilton DA, McNicol E, Babul N, Carr DB. The analgesic efficacy and safety of a novel intranasal morphine formulation (morphine plus chitosan), immediate release oral morphine, intravenous morphine, and placebo in a postsurgical dental pain model. Anesth Anal. 2008; 107: 2018-24.

Colluze PH, Schwertzberg L, Conroy JD, Charapata S, Gay M, Busch MA, Chave J, Ashley J, Lebo D, McCracken M, Portenoy RK. Breakthrough cancer pain: A rendomized trial comparing oral transmucosal fentanyl citrate and morphine sulphate immediate release. Pain 2001; 91: 123-30.

Conno FD, Ripamonti C, Fagnoni E, Brunelli C. The MERITO Study: A multicentre trial of the analgesic effect and tolerability of normal-release oral morphine during 'titration phase' in patients with cancer pain. Palliat Med. 2008; 22: 214-21

Dale O, Piribour M, Kassa S, Moksness K, Klepstad P. A double-blind, randomized, crossover comparison between single-dose and double-dose immediete-release oral morphine at bedtimein cancer patients. J Pain Symptom Manage. 2009; 37: 68-76.

Dehgan R, Ramakrichnan J, Ahmed N, Harding R. The use of morphine to control pain in advanced cancer: An investigation of clinical usage in Bangladesh. Palliat Med. 2010; 24: 707-14.

Deschamps M, Band PR, Hislop TG, Rusthoven J, Iscoe N, Warr D. The evaluation of analgesic effects in cancer patients as exemplified by a double-blind, crossover study of immediate-release versus controlled-release morphine. J Pain Symptom Manage. 1992; 7: 384-92.

Downie WW, Leatham PA, Rhind VM, Wright V, Branco JA, Anderson JA. Studies with pain rating scales. Ann Rheum Dis. 1978; 37: 378-81.

Eyelede OR, Ajayi IO, Elumelu TN, Soyannwo OA, Akinyemi OA. Oral morphine effectiveness in Nigerian patients with advanced cancer. J Pain Palliat Care Pharmacother. 2012; 26: 24-29.

Finn JW, Walsh TD, MacDonald N, Bruera E, Krebs LU, Shepard KV. Placebo-blinded study of morphine sulphate sustained-release tablets and immediate-release morphine sulphate solution in outpatients with chronic pain due to advanced cancer. J Clin Oncol. 1993; 11: 967-72.

Grassby PF, Hutchings L. Factors affecting the physical and chemical stability of morphine sulphate solutions stored in syringes. Int J Pharm Pract. 1993; 2: 39-43.

Hagen NA, Biondo P, Stiles C. Assessment and management of breakthrough pain in cancer patients: Current approaches and emerging research. Curr Pain Headache Rep. 2008; 12: 241-48.

Hanks GW, Conno FD, Cherny N, Hanna M, Kalso E, McQuay HJ, Mercadante S, Meynadier J, Poulain P, Ripamonti C, Radbruch L, I Casas J Roca, Sawe J, Twycross RG, Ventafridda V. Morphine and alternative opioids in cancer pain: The EAPC recommendation. Br J Cancer. 2001; 84: 58793.

Kamuhabwa A, Ezekiel D. Rational use and effectiveness of morphine in the palliative care of cancer patients at the Ocean Road Cancer Institute in De res salaam, Tanzania. Tanzan J Health Res. 2009; 11: 171- 74.

Khatun S, Molla MR, Akhtaruzzaman AKM, Rahman MS. Effectiveness of multimodal preemptive analgesic therapy in maxillofacial surgery. Bangladesh J Physiol Pharmacol. 2008: 24: 17-24.

Kumar KS, Rajagopal MR, Naseema AM. Intravenous morphine for emergency treatment of cancer pain. Palliat Med. 2000; $14:$ 183-88.

Lawrence AT, Quanyun A, Lien P. Physical and chemical stability of morphine sulphate $5-\mathrm{mg} / \mathrm{mL}$ and $50-\mathrm{mg} / \mathrm{mL}$ packaged in plastic syringe. Int J Pharm Compd. 2002; 6: 70- 
73.

Lee G, Sabra K, Doyle L, Doyle N, O' Malley G. Stability of morphine sulphate in PCAs. EJHP. 2003; 1-3.

Likert R. A technique for the measurement of attitudes. Arch Psych. 1932; 22: 1-55.

Melzack R, Mount BM, Gordon GM. The brompton mixture versus morphine solution given orally: Effects on pain. Can Med Assoc J. 1979; 120: 435-38.

Preechagoon D, Sumyai V, Tontisirin K, Aumpon S, Pongjanyakul T. Formulation development and stability testing of oral morphine solution utilizing preformulation approach. J Parm Pharmaceut Sci. 2005; 8: 362-69.

Quigley C, Joel S, Patel N, Baksh A, Slevin M. Plasma concentration of morphine, morphine-6-glucoronide and morphine -3-glucoronide and their relationship with analgesia and side effects in patients with cancer-related pain. Palliat Med. 2003; 17: 185- 90 .

Rahman MS. Changes required in pharmacotherapy teaching to ensure rational use of drugs. Bangladesh J Physiol Pharmacol. 1995: 11: 38-39.

Rahman MS, Kamal ASMA, Chowdhury S, Khan IA, Islam AMZ, Sultana R, Begum M, Akhter N, Anwar AKMN.
Exercise on selection of P-drug: Preliminary evaluation of a newer method of pharmacotherapy teaching in Bangladesh. Bangladesh J Physiol Pharmacol. 2000: 16: 50-54.

Rahman MS, Huda S. Antimicrobial resistance and related issues: An overview of Bangladesh situation. Bangladesh J Pharmacol. 2014: 9: 218-24.

Scott J, Huskisson EC. Vertical or horizontal visual analogue scales. Ann Rheum Dis. 1979; 38: 560.

Todd J, Rees E, Gwillium B, Davis A. An assessment of the efficacy and tolerability of a 'double dose' of normal-release morphine sulphate at bedtime. Palliat Med. 2002; 16: 507-12.

Vermeire A, Remon JP. Stability and compatibility of morphine. Int J Pharm. 1999; 187: 17-51.

World Health Organization (WHO). World Health Organization cancer pain relief with a guide to opioid availability. 2nd ed. Geneva, WHO, 1996.

Yeh SY, Lach JL. Stability of morphine in aqueous solution. III. Kinetics of morphine degradation in aqueous solution. J Pharm Sci. 1961; 50: 35-42.

Zeppetella G. Impact and management of breakthrough pain in cancer. Curr Opin Support Palliat Care. 2009; 3: 1-6. 


\section{Your feedback about this paper}

1. Number of times you have read this paper 0

2. Quality of paper Click

3. Your comments 